\title{
Risk Factors for Infectious Complications in Patients Undergoing Retrograde Intrarenal Surgery
}

\author{
Demirhan Orsan Demir11, Omer Gokhan Doluoglu1, Yildiray Yildiz1, Selen Bozkurt², Ali Ayyildiz and Arif Demirbas
}

\begin{abstract}
Objective: To investigate the risk factors that may cause urinary tract infection (UTI) in patients applied with retrograde intrarenal surgery (RIRS).

Study Design: An observational study.

Place and Duration of Study: Departments of Urology, Ankara Training and Research Hospital, Turkey, from September 2014 to April 2017.

Methodology: A retrospective examination was made of patients who underwent RIRS. The patients were separated into 2 groups as those with no UTI in the postoperative period (Group 1) and those with UTI (Group 2). The groups were compared in respect of age, stone size, operating time, presence of residual stone, and body mass index. Continuous independent variables were compared using the Student's t-test and in the comparison of categorical variables, the Chisquare test was used. A value of $p<0.05$ was accepted as statistically significant.

Results: Group 1 comprised 169 patients with no UTI and Group 2, 20 patients with UTI. The mean operating time was $55.82 \pm 14.73$ minutes in Group 1 and $75.5 \pm 23.9$ minutes in Group 2 ( $p=0.002)$. In multivariate analysis, operating time was determined as an independent prognostic risk factor increasing the risk of infection $(p=0.001)$. The cut-off value determined with ROC analysis was 61 minutes. When operating time exceeded 61 minutes, the infection risk was increased 11.1 -fold (sensitivity $75 \%$, specificity $76 \%$, AUC 0.76 ).

Conclusion: Operating time in patients applied with RIRS was determined to be an independent prognostic risk factor for UTI risk in the postoperative period. In patients where surgery lasts more than 1 hour, particular attention should be paid in respect of infection risk.
\end{abstract}

Key Words: Urinary tract infection, Retrograde intrarenal surgery, Infection risk.

\section{INTRODUCTION}

In recent years there have been significant changes in the treatment of upper urinary system stones. With the advances in endourological technology, the minimally invasive treatment approach has become more popular. ${ }^{1}$ Increased use of flexible ureterorenoscopy and holmium laser has made treatment possible of upper urinary system stones of various sizes with high stonefree rates, low morbidity, short hospital stay, and high patient comfort. 2 With these advantages, retrograde intrarenal surgery (RIRS) has become one of the most important current treatment options applied to upper urinary system stones $<2 \mathrm{~cm} .{ }^{3}$ In the European Urology Association (EUA) guidelines, it is shown together with shockwave lithotripsy (SWL) as an alternative treatment to percutaneous nephrolithotomy (PNL) for stones $>2 \mathrm{~cm}$.

1 Department of Urology, Ankara Training and Research Hospital, Ankara, Turkey

2 Department of Biostatistics and Medical Informatics, Faculty of Medicine, Akdeniz University, Antalya, Turkey

Correspondence: Dr. Demirhan Orsan Demir, Departments of Urology, Ankara Training and Research Hospital, Ankara, Turkey

E-mail:rsndmr@hotmail.com

Received: June 21, 2018; Accepted: December 06, 2018
In selected cases with negative factors such as low success of SWL in lower pole stones, ${ }^{4}$ the difficulty of applying SWL and PNL in obese patients, pregnancy or coagulopathy, RIRS has become the primary treatment choice for stones $>2 \mathrm{~cm} .{ }^{2}$ Although the treatment area of RIRS has broadened to this extent and it is preferred in many centres because of the low morbidity rates, there are also complications of RIRS, which cannot be ignored. 5

The most frequently encountered complication of this procedure is postoperative infection and despite prophylactic antibiotic treatment, incidence has been reported in literature of $1.7-18.8 \%{ }^{6}$ Just as these infective complications may be postoperative fever or urinary tract infection (UTI), they may also be urosepsis or even septic shock. ${ }^{7}$

Together with broadened indications, RIRS is currently often used in patients with a greater stone burden and related to this, the operating time is prolonged. There are many studies in literature related to RIRS and complications but to the best of our knowledge, there are relatively few studies which have examined the risk factors that facilitate postoperative infection.

The aim of this study was to investigate the risk factors that could cause postoperative infective complications in patients undergoing RIRS. 


\section{METHODOLOGY}

Data of 221 patients, who were treated with RIRS because of kidney stones at Departments of Urology, Ankara Training and Research Hospital, Turkey, from September 2014 to April 2017, were evaluated retrospectively. Approval was granted by the Local Ethics Committee before starting the study (065). A record of age, gender, body mass index (BMI), American Society of Anaesthesiologists (ASA) scores, Charlson Comorbidity Index $(\mathrm{CCl})$ values, ${ }^{8}$ congenital urinary anomalies and preoperative $\mathrm{JJ}$ stent was made for all patients. Preoperatively, all patients were evaluated with non-contrast computed tomography (CT). Data related to stones were recorded as number of stones, localisation (renal pelvis, upper/mid/lower calyx, and multiple calyceal localisations), history of previous stone surgery, stone size (maximum length of the stone and for multiple stones, the total of the maximum lengths). Infective complications were recorded according to the modified Clavien-Dindo Classification (MCDC). ${ }^{9}$

Patients with production in urine culture were treated with appropriate antibiotics, re-evaluated and then operated upon. Antibiotic prophylaxis (ceftriaxone $2 \mathrm{gr}$ intravenous) was administered routinely to all patients starting on the day of operation. 10 Preoperative urine culture was obtained from all patients. If they had infectious complications such as $>38^{\circ} \mathrm{C}$ fever, acute pyelonephritis, and sepsis postoperatively, all cultures (urine, blood, throat) were obtained from the patients. Patients were excluded from the study, if the preoperative urine culture was not sterile, if they had resistant urinary system infection, if they were applied with bilateral RIRS, and if they had hydronephrosis or chronic urinary pathology (e.g: neurogenic bladder, presence of indwelling catheter etc.).

All the procedures were performed by 2 surgeons with sufficient experience of RIRS, with the patient in the standard lithotomy position under general anaesthesia. The operating time was defined and recorded as the time from the entry of the semi-rigid ureterorenoscope into the ureter to the completion of the operation. The ureteral access sheath (UAS) (Flexor 9.5/11,5 Fr, Cook Medical Bloomington, IL, USA) was used in all patients to reduce intrarenal pressure.11 All operations were performed with non-digital flexible ureterorenoscope (Flex $\mathrm{X} 2^{\mathrm{TM}}$, Karl Storz, Tutlingen, Germany). The irrigation rate was kept below $25 \mathrm{ml} / \mathrm{min}$ and from time to time the irrigation pressure was increased depending on the image quality. Before intracorporeal lithotripsy in lower pole stones where access is difficult, the stone was moved to an appropriate calyx with a $1.9 \mathrm{Fr}$ Zero Tip nitinol basket (Boston Scientific, USA) catheter. Due to the potential ischaemic effects of UAS, all the cases were terminated before exceeding 120 minutes. ${ }^{11,12}$ In all cases, a JJ stent was placed and if there were no residual fragments, the stent was removed after 1-3 weeks.

Patients with no symptoms (fever, lateral pain, and hematuria) were discharged on postoperative day-1. Patients were followed-up with plain urinary tract $\mathrm{X}$-ray on postoperative day-1. At the end of one month, evaluations of non-contrast CT images (thickness $2.5 \mathrm{~mm}$ ) were made by a single urologist (OGD). Treatment success was considered as a stone-free status or presence of clinically insignificant residual fragments $(<4 \mathrm{~mm})$ on CT and plain urinary tract X-ray. Symptomatic residual stones were treated later with a staged procedure (stage RIRS) or with SWL.

Infective complications were defined as temperature $>38^{\circ} \mathrm{C}$, acute pyelonephritis, positive blood and urine culture and sepsis which developed in the postoperative period and continued for 48 hours. ${ }^{6}$ Sepsis which occurred because of infection was defined as systemic inflammatory response syndrome (SIRS). The SIRS criteria were defined according to American College of Chest Physicians and the Society of Critical Care Medicine in 2004.13

The patients were separated into 2 groups as those with no UTI/fever in the postoperative period (Group 1) and those with UTI/fever (Group 2). The data analysis was performed using SPSS for Windows, version 11.5 (SPSS Inc., Chicago, IL, USA). Descriptive statistics for variables with a normal distribution, non-normal distribution and categorical variables were shown as mean \pm standard deviation, median (min-max) and the number of cases and percentages (\%), respectively. The normality of the distribution was tested with P-P plot and Kolmogorov-Smirnov tests. In the comparison of continuous independent variables between the two groups, the Student's t-test and Mann-Whitney U-test were used; and in the comparison of categorical variables, the Chi-square test was used. Values determined as statistically significant in the univariate analysis were evaluated with multivariate logistic regression analysis to determine whether or not they were independent prognostic risk factors, increasing the risk of infection. A cut-off value for RIRS duration was determined with a ROC curve. A value of $p<0.05$ was accepted as statistically significant.

\section{RESULTS}

Two hundred and twenty-one patients underwent RIRS during the study. After exclusions, the data were analysed of 189 patients who met the study criteria. Group 1 comprised 169 patients with no UTI and Group 2, 20 patients with UTI. No statistically significant difference was found between the groups in respect of age $(p=0.399)$, gender $(p=0.125)$, BMI $(p=0.324)$, diabetes mellitus $(D M, p=0.92)$, and urinary anomalies $(p=0.165)$. Stone size was median $15 \mathrm{~mm}$ (range 5-80 mm) 
in Group 1 and $23.5 \mathrm{~mm}$ (range 8-56 $\mathrm{mm}$ ) in Group 2 $(p=0.004)$. In the multivariate analysis, stone size was not determined to be an independent prognostic risk factor for infection risk $(p=0.259)(95 \% \mathrm{Cl} 0.979-1.082)$. Stone localisation was classified as upper, mid, lower calyx, pelvis and multicalyceal and no statistically significant difference was determined between the two groups in respect of localisation $(p=0.515)$. No statistically significant difference was determined between the two groups in respect of whether the stone was solitary or multiple $(p=0.595$, Table I).

There was a preoperative $\mathrm{JJ}$ stent in $48(28.4 \%)$ of the Group 1 patients and in $6(30 \%)$ of the Group 2 patients, with no statistically significant difference determined between the groups $(p=0.925)$. In the analysis made according to the history of stone surgery, no difference was seen between the groups $(p=0.601)$. When patients were classified according to the $\mathrm{CCl}$, no difference was determined between the groups $(p=0.145)$. In $11(6.5 \%)$ patients in Group 1 and 5 (25\%) patients of Group 2, an ASA score of 3 was determined $(p=0.014)$. In the multivariate analysis, an ASA score of 3 was determined as an independent prognostic risk factor increasing infection risk $(p=0.036,95 \% \mathrm{Cl}=1.119-29.013)$. The descriptive data of the study are shown in Table $\mathrm{I}$.

The mean operating time was $55.82 \pm 14.73$ minutes (range, 15-100 minutes) in Group 1 and $75.5 \pm 23.9$ minutes (range, 35-120 minutes) in Group 2; and the difference between the groups was determined to be statistically significant $(p=0.002)$. In multivariate analysis, operating time was determined as an independent prognostic risk factor increasing the risk of infection $(p=0.001)(95 \% \mathrm{Cl}, 2.617-32.788)$. The cut-off value determined with ROC analysis was 61 mins. When operating time exceeded 61 mins the infection risk was increased 11.1 -fold (sensitivity $75 \%$, specificity $76 \%$, AUC 0.76).

The median postoperative length of stay in hospital was calculated as 1 day (range, 1-2 days) in Group 1 , and 4 days (range, $1-14$ days) in Group $2(p<0.001)$. At the postoperative follow-up examinations, 134 (79.28\%) patients in Group 1 were seen to be stone-free and 11 $(55 \%)$ patients in Group $2(p=0.016)$. In multivariate analysis, the presence of residual stones was not seen to be an independent risk factor increasing infection $(p=0.711)$. The perioperative and postoperative data of the patients and the multivariate analysis results are shown in Tables II and III.

When the 20 patients in Group 2 were classified according to the MCDC score, $8(40 \%)$ patients were grade 1 and $11(55 \%)$ patients were grade 2 . In $1(5 \%)$ patient, a diagnosis of sepsis was made and broad spectrum antibiotherapy was started. In the urine cultures taken from the Group 2 patients, production of Escherichia coli was determined in $5(25 \%)$ patients,
Klebsiella pneumoniae in $2(10 \%)$ patients, and Pseudomonas aeruginosa in $1(5 \%)$ patient (the patient with sepsis).

Table I: Demographic, clinical and preoperative data.

\begin{tabular}{|c|c|c|c|}
\hline & Group 1 n (\%) & Group 2 n (\%) & $p$-value \\
\hline Total number of patients & $169(89.41)$ & $20(10.59)$ & 0.125 \\
\hline Female patients & $71(42)$ & $12(60)$ & \\
\hline Male patients & $98(58)$ & $8(40)$ & \\
\hline Age (years) (mean $\pm S D$ ) & $47.09( \pm 14.2)$ & $49.95( \pm 14.2)$ & 0.399 \\
\hline $\mathrm{BDI}\left(\mathrm{kg} / \mathrm{m}^{2}\right)($ mean $\pm \mathrm{SD})$ & $27.55( \pm 3.23)$ & $26.8( \pm 3.02)$ & 0.324 \\
\hline \multicolumn{4}{|l|}{ ASA Score } \\
\hline ASA 1 & $56(33.13)$ & $7(35)$ & \\
\hline ASA 2 & $102(60.35)$ & $8(40)$ & \\
\hline ASA 3 & $11(6.5)$ & $5(25)$ & $0.014^{1}$ \\
\hline \multicolumn{4}{|l|}{ Diabetes } \\
\hline No & $145(85.8)$ & $15(75)$ & \\
\hline Yes & $24(14.2)$ & $5(25)$ & 0.923 \\
\hline \multicolumn{4}{|l|}{ Urinary anomaly } \\
\hline No & $164(97.05)$ & $20(100)$ & \\
\hline Yes & $5(2.95)$ & 0 & 0.165 \\
\hline \multicolumn{4}{|l|}{ Operating history } \\
\hline No & $91(53.8)$ & $12(60)$ & \\
\hline Yes & $78(46.2)$ & $8(40)$ & 0.601 \\
\hline $\begin{array}{l}\text { Stone size (mm) } \\
\text { (median, min-max) }\end{array}$ & $15(5-80)$ & $23.5(8-56)$ & $0.004^{1}$ \\
\hline \multicolumn{4}{|l|}{ Multiple stones } \\
\hline No & $130(66.93)$ & $15(75)$ & \\
\hline Yes & $39(23.07)$ & $5(25)$ & 0.763 \\
\hline \multicolumn{4}{|l|}{ Preoperative $\mathrm{JJ}$ stent } \\
\hline No & $121(71.6)$ & $14(70)$ & \\
\hline Yes & $48(28.4)$ & $6(30)$ & 0.925 \\
\hline
\end{tabular}

Table II: Postoperative data.

\begin{tabular}{l|c|c|c}
\hline & Group 1 (\%) & Group 2 (\%) & p value \\
\hline $\begin{array}{l}\text { Operating time } \\
\text { (mins) (mean } \pm \text { SD) }\end{array}$ & $55.82 \pm 14.73$ & $75.5 \pm 23.9$ & $0.002^{1}$ \\
\hline $\begin{array}{l}\text { Length of stay in hospital } \\
\text { (days) (median, min-max) }\end{array}$ & $1(1-2)$ & $4(1-14)$ & $<0.001$ \\
\hline $\begin{array}{l}\text { Stone-free rates } \\
\text { No }\end{array}$ & $35(20.72)$ & $9(45)$ & $0.016^{1}$ \\
Yes & $134(79.28)$ & $11(55)$ & \\
\hline
\end{tabular}

${ }^{1}$ Significant at $p<0.05$

Table III: Multivariate logistic regression analysis: Predictive factors for postoperative infectious complications.

\begin{tabular}{l|c|c|c}
\hline & $\mathrm{p}$-value & Odds ratio & $95 \% \mathrm{Cl}$ \\
\hline Operating time & $0.001^{1}$ & 11.1 & $2.167-32.788$ \\
\hline ASA 3 score & $0.036^{1}$ & 5.699 & $1.119-29.013$ \\
\hline Stone size & 0.259 & 1.029 & $0.979-1.082$ \\
\hline Residual stone & 0.711 & 1.265 & $0.365-4.389$ \\
\hline \multicolumn{4}{l|}{ Cl=Confidence interval, ASA=American Society of Anesthesiologist; ${ }^{1}$ Significant at $p<0.05$}
\end{tabular}

\section{DISCUSSION}

With the application of RIRS, treatment of upper urinary system stones has become possible with high stone-free rates and low morbidity. ${ }^{2}$ RIRS has been shown to be an alternative to PNL in selected cases. ${ }^{10}$ There is currently increasing use of this method in stones $>2 \mathrm{~cm}$. Together with technological advances, minimally invasive surgical 
approaches are being increasingly preferred by clinicians and patients. In industrial societies, in particular, if the importance of return to work in the shortest possible time after surgery is taken into consideration, the short hospitalisation duration of RIRS is attractive.1,14 However, in a procedure which has become so popular, it is important to predict which factors increase the risk of postoperative urinary system infection.

Despite prophylactic antibiotic treatment, the incidence of UTI after RIRS has been reported as $1.7 \%-18.8 \%$ in literature.1,5-7 Similarly, in the current series, this rate was found to be $10.58 \%$. As reported by Fan et al., one of the reasons for an increase in postoperative infection risk is an increase in stone size. ${ }^{6}$ In the current study, stone size was also found to be related to postoperative infection. Moreover, an increase in stone size is the most significant factor prolonging operating time. 15 There are studies in literature that have reported that prolonged operating time is a risk factor for postoperative fever and in those studies, the operating time has been reported as $60-120$ minutes.5,6,16,17 In the multivariate analysis of the current study, operating time was determined to be an independent prognostic risk factor, increasing the risk of infection $(p=0.001)$. The cutoff value determined with $\mathrm{ROC}$ analysis was found to be 61 minutes. An increase of operating time beyond 61 minutes increased the infection risk by 11.1-fold. When compared with the above-mentioned studies, the cut-off value of the operating time in the current study was found to be similar to that of Xu et al. 5 In a study by Alezra et al. that included 243 patients with kidney stones and 23 patients with collecting system tumour, postoperative infection was seen to be more frequent following operations that lasted longer than 70 minutes, and this duration was close to the value in the current study. ${ }^{17}$ Although, no definitive safe operating time has been determined, a lengthy RIRS duration is a risk factor for postoperative fever/infection, which must be taken into consideration in patients with the other risk factors mentioned below.

As there are studies in literature that have shown a relationship between the presence of multiple stones and postoperative infective complications, there are also studies that have not determined such a relationship.1,7 In the analysis of the current study, the rates of multiple stones were similar in both groups. The completely stone-free rate in the current study was calculated as $76.6 \%$ and this was similar to findings in literature. Stone size is known to be a predictor of residual stones. ${ }^{18}$ In the current study, there was seen to be a relationship between the presence of residual stone and infective complications and this was consistent with other studies in literature.7,19 It can be seen from previous studies that the rates of residual stones are increased in lower pole stones. In a study by Resorlu et al., a relationship was found between stone localisation and stone-free rates; and Cohen et al. reported that the highest rate of failure was in lower pole stones and staghorn stones.20,21 Taking this into consideration in the current study, residual fragments in the lower pole were removed from the kidney with a basket catheter as much as possible. Moreover, in cases where the stone in the lower pole could not be reached easily, the stone was moved to a suitable calyx with a basket catheter, as far as the stone burden allowed and was then fragmented. In the current study, no relationship was seen between stone localisation and infective complications. This can be attributed to clearing residual fragments of lower pole stones with the basket catheter and moving lower pole stones that could not be easily accessed to another calyx. Similarly, Bas et al. determined no relationship between stone localisation and complications. ${ }^{1}$

Urinary anomaly is one of the reasons increasing the risk of postoperative complications in patients applied with RIRS.20,22 In the current series, only five patients had urinary anomalies (horseshoe kidney) and no infection was seen in any of these cases. As the number of patients with urinary anomaly was low, no strong interpretation can be made on this subject.

An ASA score of 3 in the current study was seen to be an independent prognostic risk factor for postoperative infective complications. Similarly, in literature there are studies showing that a high ASA score is a risk factor for re-presentation after discharge and for postoperative infection.7,14 Although some studies in literature have shown high rates of patients with a $\mathrm{CCl}$ score of $\geq 2$ re-presenting after discharge and being readmitted to hospital, the $\mathrm{CCl}$ scores in the current study were similar in both groups. ${ }^{14}$

When literature was examined in respect of the effect of a history of stone surgery on infective complications, there were seen to be studies showing that a history of stone surgery was a risk factor for infective complications; and there were studies where previous surgery was not seen as a risk factor.6,7 In the analysis of the current study, previous renal stone surgery was not found to be a risk factor for infective complications.

There were some limitations to the current study. First, it was retrospective so there was no analysis of the stones or reporting of the stones in Hounsfield units. Second, the study was conducted in a single centre. Nevertheless, all the procedures were performed by two experienced surgeons, which are strong aspects of the study.

RIRS is an effective and safe method which is currently routinely used in the treatment of renal stones. Although the rates of major complications are low, infective complications are seen at a rate that cannot be ignored, and these increase the length of stay in hospital.

\section{CONCLUSION}

A prolonged operating time was found to be an independent risk factor for postoperative fever/infection; 
and in cases where the time exceeded 61 minutes, this risk was increased 11-fold. Therefore, whenever possible the procedure should not exceed one hour. Another independent risk factor was an ASA score of 3 or more. Although there was a statistically significant difference between the two groups in respect of stone size and the presence of residual stones, in the multivariate analysis these were not seen to be independent risk factors for postoperative infection.

\section{REFERENCES}

1. Bas O, Tuygun C, Dede O, Sari S, Çakici MÇ, Öztürk U, et al. Factors affecting complications rates of retragrade flexible ureterorenoscopy: Analysis of 1571 procedures - a singlecenter experience. World J Urol 2017; 35:819-26.

2. Berardinelli F, Proietti S, Cindolo L, Pellegrini F, Peschechera R, Derek $\mathrm{H}$, et al. A prospective multicenter European study on flexible ureterorenoscopy for the management of renal stone. Int Braz J Urol 2016; 42:479-86.

3. Zheng $\mathrm{C}$, Xiong $\mathrm{B}$, Wang $\mathrm{H}$, Luo J, Zhang $\mathrm{C}$, Wei $\mathrm{W}$, et al. Retrograde intrarenal surgery versus percutaneous nephrolithotomy for treatment of renal stones $>2 \mathrm{~cm}$ : A meta-analysis. Urol Int 2014; 93:417-24.

4. Pardalidis NP, Andriopoulos NA, Sountoulidis P, Kosmaoglou EV. Should percutaneous nephrolithotripsy be considered the primary therapy for lower pole stones? J Endourol 2010 24: 219-22.

5. Xu Y, Min Z, Wan SP, Nie H, Duan G. Complications of retrograde intrarenal surgery classified by the modified Clavien grading system. Urolithiasis 2018; 46:197-202.

6. Fan S, Gong B, Hao Z, Zhang L, Zhou J, Zhang Y, et al. Risk factors of infectious complications following flexible ureterorenoscope with a holmium laser: A retrospective study. Int $J$ Clin Exp Med 2015; 8:11252-9.

7. Berardinelli F, Francesco PD, Marchioni M, Cera N, Proietti S, Hennessey D, et al. Infective complications after retrograde intrarenal surgery: A new standardized classification system. Int Urol Nephrol 2016; 48:1757-62.

8. Charlson M, Wells MT, Ullman R, King F, Shmukler C. The Charlson comorbidity index can be used prospectively to identify patients who will incur high future costs. PLOS One 2014; 9:e112479.

9. Tefekli A, Ali Karadag M, Tepeler K, Sari E, Berberoglu Y, Baykal M, et al. Classification of percutaneous nephrolithotomy complications using the modified Clavien grading system: looking for a standard. Eur Urol 2008; 53:184-90.

10. Guidelines on Urolithiasis; European Association of Urology Guidelines 2017.

11. Breda A, Territo A, López-Martínez JM. Benefits and risks of ureteral access sheaths for retrograde renal access. Curr Opin Urol 2016; 26:70-5.

12. Delvecchio FC, Auge BK, Brizuela RM, Weizer AZ, Silverstein AD, Lallas $C D$, et al. Assessment of stricture formation with the ureteral access sheath. Urology 2003; 61:518-22.

13. Levy MM, Fink MP, Marshall JC, Abraham E, Angus D, Cook D, et al. 2001 SCCM/ESICM/ACCP/ATS/SIS international sepsis definitions conference. Crit Care Med 2003; 31:1250-6.

14. Buldu I, Tepeler A, Karatag T, Ozyuvali E, Elbir F, Yordam M, et al. Which factors affect the hospital re-admission and rehospitalization after flexible ureterorenoscopy for kidney stone? World J Urol 2015; 34:1291-5.

15. Sorokin I, Grau DK, Rehfuss A, Birney A, Stavrakis C, Leinward G, et al. Stone volume is best predictor of operative time required in retrograde intrarenal surgery for renal calculi: implications for surgical planning and quality improvement. Urolithiasis 2016 44:544-50

16. Takazawa R, Kitayama S, Tsujii T. Successful outcome of flexible ureteroscope with holmium lazer lithotripsy for renal stones $2 \mathrm{~cm}$ or greater. Int J Urol 2012; 19:264-7.

17. Alezra E, Lasselin J, Forzini T, François T, Viart L, Saint F. Prognostic factors for severe infection after flexible ureterorenoscopy: Clinical interest of urine culture the day before surgery? Prog Urol 2016; 26:65-71.

18. Yamashita S, Kohjimoto Y, Iba A, Kikkawa K, Hara I. Stone size is a predictor for residual stone and multiple procedures of endoscopic combined intrarenal surgery. Scand J Urol 2017; 51:159-64.

19. Dincel N, Resorlu B, Unsal A, Tepeler A, Silay MS, Armagan A, et al. Are small residual stone fragments really insignificant in children? J Pediatr Surg 2013; 48:840-4.

20. Resorlu B, Unsal A, Gulec H, Öztuna D. A new scoring system for predicting stone-free rate after retrograde intrarenal surgery: The "resorluunsal stone score". Urology 2012; 80:512-8.

21. Cohen J, Cohen S, Grasso M. Ureteropyeloscopic treatment of large, complex intrarenal and proximal ureteral calculi. BJU Int 2013; 111:E127-31.

22. Atis G, Resorlu B, Gurbuz C, Arikan O, Ozyuvali E, Unsal A, et al. Retrograde intrarenal surgery in patients with horseshoe kidneys. Urolithiasis 2013; 41:79-83. 